\title{
Mitogen-Activated Protein Kinases and Mitogen-Activated Protein Kinase Phosphatases in Regenerative Myogenesis and Muscular Dystrophy
}

\author{
Hao Shi ${ }^{1}$ and Anton M. Bennett ${ }^{1,2}$ \\ ${ }^{1}$ Department of Pharmacology \\ 2Program in Integrative Cell Signaling and Neurobiology of Metabolism, \\ Yale University School of Medicine, \\ United States
}

\section{Introduction}

The mitogen-activated protein kinase (MAPK) signal transduction pathway is required to promote skeletal myogenesis and maintain skeletal muscle function. Although it has been long appreciated that the MAPK pathway plays a critical role in skeletal myogenesis it is still unclear as to whether the MAPKs are involved in the development of skeletal muscle diseases such as muscular dystrophies. Much evidence has demonstrated that MAPK activation is important for skeletal myogenesis. The cessation of MAPK activity is also an important part of the process of skeletal myogenesis. The MAPK phosphatases (MKPs) are responsible for inactivating the MAPKs. The role of the MKPs in physiological and pathophysiological functions of skeletal muscle remains to be fully understood. In this review, we will summarize the current state of understanding of the functional roles of the MAPKs, and the emerging role of the MKPs in the regulation of physiological skeletal muscle function, and their potential involvement in skeletal muscle diseases.

\section{Mitogen-activated Protein Kinase signaling and myogenesis}

The mitogen-activated protein kinase (MAPK) cascade plays an essential role in conveying extracellular signals from growth factors, stress, and cytokines into biological responses that include differentiation, proliferation, apoptosis and cell motility (Cuevas et al., 2007; Pearson et al., 2001). Up until now, at least 4 MAPK signaling pathways have been identified: 1) extracellular signal-regulated kinase 1 and 2 (ERK1/2), 2) p38a/ $\beta / \gamma / \delta$ MAPK, 3) c-Jun $\mathrm{NH}_{2}$-terminal kinases 1, 2, and 3 (JNK1/2/3) and 4) ERK5 (Bogoyevitch, 2006; Bogoyevitch and Court, 2004; Whitmarsh, 2006; Zarubin and Han, 2005). MAPKs when phosphorylated on their regulatory threonine and tyrosine residues by their upstream MAPK kinases become activated. Once activated, these MAPKs phosphorylate substrates that include transcription factors, phospholipases, protein kinases and cytoskeletal proteins (Johnson and Lapadat, 2002; Turjanski et al., 2007). 
A large body of data suggests that the p38 MAPK pathway is pro-myogenic. p38 MAPK promotes myogenesis through 1) interaction with transcription factors. At the early stage of differentiation, p38 MAPK phosphorylates E-protein E47, which dimerizes with the transcription factor MyoD to activate muscle-specific gene expression (Lluis et al., 2005). Whereas, at later stages of differentiation, p38 MAPK phosphorylates the transcription factor MRF4 thereby repressing its transcriptional activity (Suelves et al., 2004). 2) phosphorylation of the SWI/SNF chromatin-remodeling complex, recruiting this complex to myogenic loci (Simone et al., 2004); 3) phosphorylation of the transcription factor myocyte enhancer factor-2 (MEF2) thereby enhancing its transcriptional activity (Black and Olson, 1998; Zetser et al., 1999); 4) stabilization of myogenic mRNA by directly phosphorylating KSRP, an important factor for decaying AU-rich element mRNA, and compromising its function to promote mRNA decay (Briata et al., 2005). These observations provide strong mechanistic insight into how p38 MAPK couples to the myogenic machinery.

Evidence derived from mouse models also supports the functional role of p38 MAPK in muscle differentiation (Perdiguero et al., 2007). In an effort to dissect the role of different p38 MAPK isoforms in myogenesis, Perdiguero et al used p38 $\alpha$, p38 $\beta$, p38 $\gamma$, and p38 $\delta$-deficient mice and analyzed the function of each. p38 $\alpha$ rather than p38 $\beta$ and p38 $\gamma$-deficient myoblasts failed to form multinucleated myotubes, whereas p38 $\delta$-deficient myoblasts exhibited attenuated differentiation (Perdiguero et al., 2007). Although a wide body of evidence supports the pro-myogenic role of p38 MAPK, several reports also imply the involvement of p38 MAPK in muscle cell proliferation. The concomitant activation of $\mathrm{p} 38 \mathrm{MAPK} \alpha / \beta$ and satellite cells imply that p38 MAPK may also be involved in satellite cell activation, since blockade of p38 MAPK by pharmacological inhibitors of p38 MAPK prevents both satellite cell proliferation and differentiation (Jones et al., 2005; Shi et al., 2010). A recent study using p38 $\gamma$-deficient mice revealed that muscles lacking this isoform of p38 MAPK contain 50\% less satellite cells, and these cells exhibit reduced proliferation (Gillespie et al., 2009) implying that this p38 MAPK isoform may also be an important regulator of satellite cell deposition and proliferation.

Although a critical role for ERK1/2 in satellite cell proliferation is established, surprisingly the role of ERK1/2 in myogenesis has not been well defined. Conflicting data from various groups suggest that this pathway may be tailored to respond to distinct cellular and extracellular conditions. Using 10T1/2 fibroblasts, Gredinger el showed that MEK1 and/or Raf1 positively regulates myogenesis by enhancing MyoD transcriptional activity, addition of the MEK inhibitor PD098059 represses MyoD-responsive genes (Gredinger et al., 1998). PD098059 also partially inhibits the formation of multinucleated myotubes in C2 myoblasts (Gredinger et al., 1998). In contrast, others have reported a negative effect of ERK1/2 in the regulation of myogenesis (Dorman and Johnson, 1999; Weyman and Wolfman, 1998). In 23A2 and C2C12 myoblasts, IGF-1 and FGF-2 inhibit myoblast differentiation through ERK1/2 signaling as PD098059 blocked this effect (Kontaridis et al., 2002; Weyman and Wolfman, 1998). Persistent activation of Raf/MEK/ERK1/2 by overexpression of a constitutively active Raf inhibits the fusion of embryonic chick myoblasts into multinucleated myotubes. This inhibition can be rescued by addition of PD098059 (Dorman and Johnson, 1999). Yet there is another layer of regulation of Ras/Raf/ERK1/2 on myogenesis. A novel regulator of the Ras-Raf interaction, named DA-Raf, lacks the Raf kinase domain and interferes with the binding of Ras to other targets. It was found that DA- 
Raf serves as a positive regulator of myogenic differentiation (Yokoyama et al., 2007). A recent study revealed that Grb2-associated binder 1 (Gab1) interacts with the protein tyrosine phosphatase SHP-2 to activate downstream ERK signaling, thereby inhibiting IGF-1-mediated myogenic differentiation (Koyama et al., 2008). These results are consistent with other data in which conditional deletion of SHP-2 in skeletal muscle impairs skeletal muscle growth (Fornaro et al., 2006). Taken together, these findings tend to support the notion that ERK1/2 signaling promotes myoblast proliferation and inhibits myogenic differentiation. However, further genetic data using ERK-deficient mice needs to be provided to fully conclude the relevance of ERK1/2 in myoblast proliferation and differentiation.

ERK5 is a novel member of the MAPK family and its physiological function in myogenesis remains to be fully defined. ERK5 is enriched in skeletal muscle, it is activated upon myogenic differentiation, and anti-sense RNA to ERK5 blocks entry into myogenesis (Dinev et al., 2001). A recent study revealed an essential role of ERK5 in muscle cell fusion through the transcription factors Sp1 and Klf2/4 (Sunadome et al., 2011). ERK5 has also been shown to be responsible for muscle cell fusion without interference with other differentiation processes (Sunadome et al., 2011).

Although the JNK pathway has been implicated in myoblast proliferation (Perdiguero et al., 2007) its role in myogenic differentiation remains controversial. JNK has been demonstrated to be either dispensable or negative for myogenesis (Gallo et al., 1999; Khurana and Dey, 2004; Meriane et al., 2002). It would be extremely informative if myoblasts derived from mice lacking either of the JNK isoforms were analyzed for their effects on cell proliferation and differentiation to resolve these issues.

\section{Duchenne muscular dystrophy}

The most common form of muscular dystrophy is Duchenne muscular dystrophy (DMD) which affects up to 1:3,500 males in the United States (Porter, 2000). The regenerative capacity of skeletal muscle in DMD-stricken patients is impaired due to the loss of dystrophin (Davies and Nowak, 2006). DMD patients lose muscle strength and mobility and the disease often results in death. There is neither a cure, nor an effective treatment for DMD, or similar skeletal muscle degenerative diseases (Bhatnagar and Kumar, 2010; Tedesco et al., 2010). DMD is caused by the loss or partial deficiency in the dystrophin protein, which serves as a critical component of the dystrophin glycoprotein complex (DGC) linking the cytoskeleton of the muscle fibers to that of the extracellular matrix. The loss of dystrophin cripples the functionality of the DGC rendering the muscle fiber more susceptible to stress-induced injury. Although the primary defect of DMD is the loss of dystrophin, there are multiple secondary events that contribute to the progression of the disease. These include profound inflammatory responses, extracellular matrix degradation and fibrosis. Strategies therefore that curtail some of these secondary responses have been considered as potential therapeutic avenues to treating the progression of the disease in DMD patients.

Although the DGC and its components such as dystrophin appear to primarily serve structural roles to couple the muscle fiber to the extracellular matrix, there is clearly an important intracellular role played by providing a platform from which signaling pathways are launched. These links to downstream pathways suggest that the DGC engages active 
signaling in order to regulate muscle fiber function. Hence, DGC dysregulation may lead to alterations in intracellular signaling cascades, which may contribute to the pathogenesis of the muscular dystrophies. In this regard, understanding the signaling pathways such as the MAPKs in skeletal muscle function and muscular dystrophy may provide important insight into new avenues of therapies for these diseases.

\section{MAPKs and Muscular dystrophy}

A link between the MAPKs and muscular dystrophy has been indirectly suggested by the fact that the DGC not only functions as a mechanical infrastructure to stabilize skeletal muscle cell membranes, but it also serves as a bridge between stimuli from the extracellular matrix and intracellular signaling through physically interacting with distinct proteins (Rando, 2001). For example, Grb2, an adapter protein involved in MAPK signal transduction and cytoskeletal organization, interacts with $\beta$-dystroglycan at the C-terminal proline-rich domains (Yang et al., 1995). Furthermore, $\beta$-dystroglycan can physically interact with MAPK kinase 2 (MEK2) and its downstream kinase ERK1/2 in a yeast two-hybrid screen (Spence et al., 2004). These findings imply that MAPK signaling may play an important role in the mechano- and signal transduction of extracellular stimuli to intracellular biological responses that control muscle fiber viability. However, the reports regarding the activity of the MAPKs in the pathogenesis of muscular dystrophy remain inconclusive and vary in different experimental settings. For example, it has been reported that JNK1 is highly activated in a mouse model of DMD ( $m d x$ mouse) and compound intercrosses between an $m d x$ mouse and a MyoD-deficient mouse $\left(m d x / \mathrm{MyoD}^{-/}\right)$contributes to the progressive dystrophinopathy without appreciable changes in either ERK1/2 or p38 MAPK activities (Kolodziejczyk et al., 2001). In contrast, stable over-expression of the JNK1-specific upstream kinase MKK7 disrupts the formation of myotubes in $\mathrm{C} 2 \mathrm{C} 12$ skeletal myoblasts and $\mathrm{H} 9 \mathrm{C} 2$ cardiac myoblasts (Kolodziejczyk et al., 2001). Adenoviral infection of the JNK1 specific inhibitor JIP1 (JNK interacting protein) increased the diameter of myofibers (Kolodziejczyk et al., 2001), suggesting that the MAPKs can affect the structural integrity of the myofiber. In an attempt to test whether loss of dystrophin causes aberrant mechanotransduction, Kumar et al measured the activity of the MAPKs following stretching of $m d x$ and wild type diaphragm muscles. ERK1/2, but not JNK or p38 MAPKs were significantly activated in the muscles derived from $m d x$ mice (Kumar et al., 2004). In addition, the downstream effector of ERK1/2, AP-1 was highly up-regulated (Kumar et al., 2004). In another exercise model, $m d x$ mice were subjected to treadmill exercise, p38 MAPK and ERK1/2, but not JNK1 were highly elevated in $m d x$ cardiac muscles in comparison with wild type muscles (Nakamura et al., 2002). Elevated p38 MAPK was also observed in utrophin-dystrophin double knock-out cardiac muscles (Nakamura et al., 2001). Together, these findings suggest that MAPK signaling is likely involved in the pathogenesis of muscular dystrophy, but to what extent and how exactly MAPK contributes at the molecular level to the pathogenesis of DMD remains to be established.

\section{MAPK phosphatases in skeletal muscle function and muscular dystrophy}

Equally important as the activation of the MAPKs is their inactivation, which is catalyzed by the MKPs. The MKPs belong to a sub-class of protein tyrosine phosphatases known as the dual-specificity protein phosphatases (DUSP) (Boutros et al., 2008; Soulsby and Bennett, 
2009; Tonks, 2006). The DUSPs are characterized by a consensus signature motif represented by $\mathrm{HC}(\mathrm{X})_{5} \mathrm{R}$ which defines the active site of these enzymes (Soulsby and Bennett, 2009; Tonks, 2006). MKPs inactivate the MAPKs by directly dephosphorylating the MAPKs on its regulatory threonine and tyrosine residues. The MKPs share largely the same structure comprising of a cdc 25 homology domain and a MAPK binding domain in the $\mathrm{NH}_{2}$ terminus and a $\mathrm{COOH}$-terminus PTP catalytic domain. The $\mathrm{NH}_{2}$ terminus of the MKPs controls MAPK binding and sub-cellular targeting (Wu et al., 2005), both of these attributes contribute to MAPK signaling specificity. In this regard, although the MKPs dephosphorylate the MAPKs they do so with varying degrees of potency that depends upon both their MAPK binding affinity and sub-cellular localization. There are 10 catalytically active members in this group and they exhibit distinct sub-cellular localization, responses to extracellular stimuli, tissue distribution and affinity to their substrates (Boutros et al., 2008; Groom et al., 1996; Ishibashi et al., 1994; Misra-Press et al., 1995; Muda et al., 1997; Noguchi et al., 1993; Rohan et al., 1993).

Binding of MKPs to their MAPK substrates increases phosphatase activity (Camps et al., 1998a; Hutter et al., 2000; Slack et al., 2001) and this is due to stabilization of the active enzyme-substrate complex (Field et al., 2000). MKP-3 exhibits high fidelity to its substrate ERK1/2 and upon binding its catalytic activity is enhanced (Camps et al., 1998b). Despite the fact that the MKPs dephosphorylate a common pool of MAPKs these enzymes exhibit remarkably unique physiological effects (Chi et al., 2006; Christie et al., 2005; Wu et al., 2006). Studies from MKP knock-out mice provide convincing genetic evidence to support the notion that these MKPs function in distinct ways (Nunes-Xavier et al., 2011). The complexity of the signaling pathways and biological responses that the MKPs are involved with strongly suggest that these enzymes serve as central players in the regulation of the MAPKs. Therefore, the MKPs, which have the capacity to regulate multiple MAPKs simultaneously, represent a critical signaling node of MAPK convergence. Molecules that act as signalling (before nodes) in signal transduction can be defined as those which represent a point of convergence of multiple pathways, and one that is represented by several isoforms that are both positively and negatively involved in divergent signaling. We propose that the MKPs satisfy these criteria and constitute a critical signaling node in the MAPK pathway. Given the established role of the MAPKs in skeletal myogenesis, the actions of the MKPs as critical signaling nodes of the MAPKs is likely to make them important players in this system.

MKPs in skeletal myogenesis and skeletal muscle function. Studies of the MKPs in myogenesis and skeletal muscle function remain mainly an uncharted area. Much of the work on the MKPs in skeletal muscle function has focused on the role of MKP-1. The first MKP to be implicated in skeletal muscle function was MKP-1 (Bennett and Tonks, 1997). MKP-1 is a ubiquitously expressed, nuclear localized dual-specificity phosphatase, whose substrates include predominantly p38 MAPK, JNK and to a lesser extent, ERK1/2 (Boutros et al., 2008; Owens and Keyse, 2007). MKP-1 is an immediate-early gene and is induced by numerous stresses (Owens and Keyse, 2007). Initial reports demonstrated that MKP-1deficient mice exhibit an unremarkable phenotype, suggesting that the MKPs largely serve redundant physiological roles (Dorfman et al., 1996). However, we have shown that mice lacking MKP-1 exhibit enhanced ERK1/2, JNK and p38 MAPK activities in skeletal muscle, as well as in other tissues, demonstrating that MKP-1 plays an essential physiological role as 
a negative regulator of the MAPKs (Wu et al., 2006). The earliest suggestion that MKP-1, and hence the MAPKs, participate in myogenic regulation emerged from studies in which conditional overexpression of MKP-1 was shown to stimulate precocious myogenesis in the context of the inhibitory actions of growth factors (Bennett and Tonks, 1997). MKP-1 expression levels in proliferating myoblasts are initially high, at levels presumably sufficient to allow cell proliferation but not differentiation, and declines upon the onset of myogenesis, suggesting that extinguishing the expression of MKP-1 might be a prerequisite for myogenic entry and/or progression (Bennett and Tonks, 1997; Kondoh et al., 2007; Perdiguero et al., 2007). Consistent with this, overexpression of MKP-1 when myoblasts have become irrevocably committed to myogenesis inhibits multinucleated myotube formation (Bennett and Tonks, 1997; Kondoh et al., 2007). Hence, MKP-1 plays both positive and negative roles in myogenesis in a temporal manner by selectively regulating one or more MAPKs (Figure 1). MKP-1 appears to be directly coupled to the myogenic transcriptional machinery as studies have shown that MKP-1 is a target for upregulation by MyoD (Shi et al., 2010). Within the proximal promoter of MKP-1 there resides an E-box binding site that serves to mediate MKP-1 activation by MyoD (Shi et al., 2010). Hence, upon the initiation of myogenesis the activation of MyoD leads to an initial upregulation of MKP-1, which may be required to inactivate ERK1/2 and thus facilitate cell cycle exit in the transition towards myogenic entry. Later on during myogenesis $\mathrm{MyoD}$ was shown to uncouple from the MKP1 promoter and hence downregulate MKP-1 expression (Figure 1). Downregulation of MKP1 during the later stages of myogenesis may facilitate the increased p38 MAPK activation, which is important for multinucleated myotube formation. As such, the complexity of the outcome through which MKP-1 integrates multiple MAPK activities cannot be simply inferred by the implied actions of a single MAPK family member. These results using cultured myoblast cell lines are supported by in vivo data where it has been shown that regenerative myogenesis in response to cardiotoxin-induced injury is impaired in MKP-1deficient mice (Shi et al., 2010). These results support the notion that MKP-1 is an important regulator of myogenesis.

MKP-1 is also implicated in adult skeletal muscle fiber specialization (Shi et al., 2008). Overexpression of MKP-1 in adult type IIb (glycolytic) myofibers converts these fibers to slower-twitch type IIa or type I (oxidative) fibers, suggesting that MKP-1-mediated dephosphorylation of MAPK signaling is required to maintain the glycolytic fiber phenotype through the repression of slow myofibers (Shi et al., 2008). Consistent with these data, it has been shown that MKP-1-deficient mice are protected from the loss of oxidative myofibers during high fat diet-induced obesity (Roth et al., 2009). Hence, decreased MKP-1 expression results in enhanced MAPK signaling, which protects from the loss of glycolytic myofibers by driving oxidative myofiber conversion. The mechanistic basis for these data is based upon the observation that MKP-1 mediates p38 MAPK phosphorylation of the peroxisome proliferator-activated receptor $\gamma$ co-activator $1 \alpha$ (PGC-1 $\alpha$ ) (Roth et al., 2009), which is required to promote oxidative myofiber conversion. Collectively, these results suggest that MKP-1 plays an essential role in the maintenance of glycolytic/oxidative myofiber composition. MKP-1 is also suggested to be involved in the maintenance of muscle mass (Shi et al., 2009). Overexpression of MKP-1 in slow-twitch soleus muscles and in fasttwitch gastrocnemius muscles reduces muscle fiber size, though this reduction in fiber size may go through distinct molecular mechanisms (Shi et al., 2009). 
MYOTRAUMA

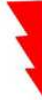

RELEASE OF

Growth Factors (e.g. FGF's)

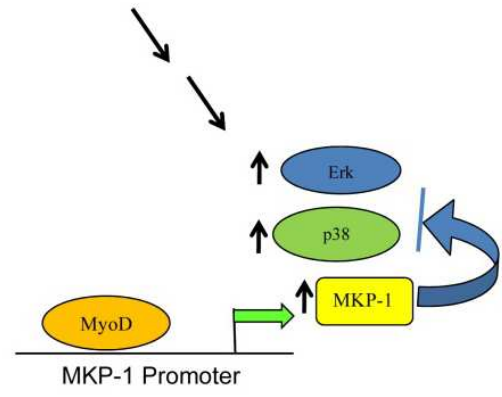

LOW MAPK Activity

[Proliferation Permissive]

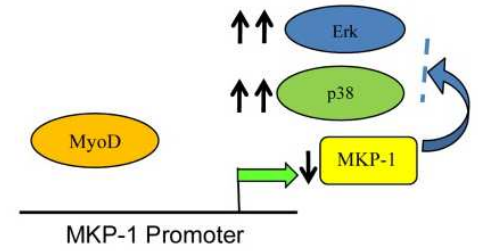

HIGH MAPK Activity

[Differentiation Permissive]

Fig. 1. MAPK/MKP signaling coordinates myogenesis. Following myotrauma the MAPKs become activated to drive cell proliferation. MKP-1 is upregulated by these MAPKs and MyoD. MKP-1 sets the threshold of MAPK activity that permits myoblast proliferation [Proliferation Permissive] but not differentiation. Upon the initiation of differentiation, MyoD uncouples from the MKP-1 promoter causing its expression levels to fall, thereby removing the inhibitory actions of MKP-1 on the MAPKs. This allows for higher levels of MAPK activity to be achieved, in particular p38 MAPK, which promotes differentiation [Differentiation Permissive].

MKP-1 also plays a regulatory role in estrogen-related receptor $\alpha(E R R \alpha)$ and PGC-1 $\alpha$ mediated myogenic differentiation. Direct up-regulation of MKP-1 by ERR $\alpha$ and PGC-1 $\alpha$ at the early stage of myogenesis inactivate ERK1/2 signaling and facilitate the progression of myogensis as MEK inhibition rescues the myogenic defect in ERRa-/- myoblasts (Murray and Huss, 2011). Recent work from this laboratory suggests that other MKPs, in addition to MKP-1, also contribute to the regulation of skeletal muscle function. Mice lacking MKP-5, which interestingly also dephosphorylates predominately p38 MAPK and JNK, exhibit enhanced skeletal muscle regeneration distinct from that observed with MKP-1-deficient mice (H.S. and A.M.B., unpublished observations). These results suggest that the MKPs might play distinct roles in not only coordinating myogenic activation and progression but they may do so through specific and non-overlapping mechanisms.

A role for MKP-1 in muscular dystrophy. The generation, maintenance, and repair of adult skeletal muscle is critically dependent upon the activation and self-renewal of satellite cells (Wagers and Conboy, 2005). In response to skeletal muscle injury, myofiber-released growth factors and cytokines stimulate satellite cell proliferation, migration and differentiation by activating signaling cascades including the MAPK pathway. It is thought that the depletion of satellite cells during the progression of DMD is a major factor that precipitates the 
ultimate failure of muscle function. Therefore, by modulating satellite cell activation, differentiation and/or self-renewal DMD can theoretically be improved.

Several reports have addressed the role of the MAPKs in $m d x$ mice, however there is no underlying consensus as to whether the MAPKs are definitively involved in the pathogenesis of the dystrophic phenotype. Some reports show upregulation of ERK1/2, JNK2 and p38 MAPK (Nakamura et al., 2005), whereas others have shown a downregulation of p38 MAPK and an upregulation of ERK1/2 (Lang et al., 2004), yet others find no consistent differences in p38 MAPK (Nakamura et al., 2001). To study the pathophysiological role of MKP-1 in Duchenne muscular dystrophy, we inter-crossed MKP1 knockout mice into the $m d x$ background in order to determine whether loss of MKP-1 ameliorates or exacerbates the dystrophic phenotype. The advantage of generating an MKP1-deficient animal model is that instead of studying an individual MAPK, we examined the integration of several MAPKs that become hyperactivated due to the lack of MKP-1. We found that $m d x / m k p-1 \%$ mice have reduced body weight and muscle mass in comparison with $m d x / m k p-1^{+/+}$mice (Shi et al., 2010). The reduction of body weight may be attributed to the chronic elevated levels of inflammation or it is also likely that this is due to an underlying metabolic defect that we have observed in mice lacking MKP-1 that is related to increased energy expenditure (Roth et al., 2009; Wu et al., 2006). Histological analysis of muscle sections from $m d x / m k p-1 \%$ mice revealed that MKP-1 deficiency exacerbates the pathogenesis of muscular dystrophy (Shi et al., 2010). This exacerbation may be accounted for by a combination of two factors that are cell autonomous and/or directly related to defects in satellite cell function as well as a contribution from a hyperactivated immune response. Satellite cells from MKP-1-deficient muscles exhibit reduced proliferative capacity whereas precocious differentiation was evident even under high serum conditions (Shi et al., 2010). Additionally, increased levels of macrophage and neutrophil infiltrates into damaged myofibers in $m d x / m k p-1^{-}$mice compared to MKP-1 wild type $m d x$ mice, this was observed along with serum and skeletal muscle cytokine levels that are significantly increased in $m d x / m k p-1 \%$ mice (Shi et al., 2010). Collectively, these findings suggest that MKP-1 is critical for the regulation of muscle regeneration in DMD by modulating both immune responses and satellite cell proliferation and differentiation (Figure 2). Further studies employing conditional deletion of MKP-1 in the satellite cell and hematopoietic compartments will be required in order to determine the contribution of MKP-1 in these tissues to the overall skeletal muscle regenerative defect.

\section{Therapeutic targeting of MAPK/MKPs in muscular dystrophy}

Research on MKP-1 and its involvement in regenerative myogenesis and muscular dystrophy suggests that MKP-1 may play an important role in the progression of muscular dystrophy and possibly other degenerative skeletal muscle diseases. Therefore, targeting the MAPK/MKP signaling pathway in order to ameliorate skeletal muscle disease and specifically, muscular dystrophy, merits further investigation. However, definitive validation that the MAPK/MKP module is a valid therapeutic target for muscular dystrophy is still lacking. There has been some suggestion that interference with the MAPK/MKP signaling module may have therapeutic value. It has been shown that adenoviral delivery of the JNK1 inhibitory protein, JIP1, can attenuate the pathogenesis of dystrophic fibers (Kolodziejczyk et al., 2001), implying that inhibition of JNK1 may serve as 


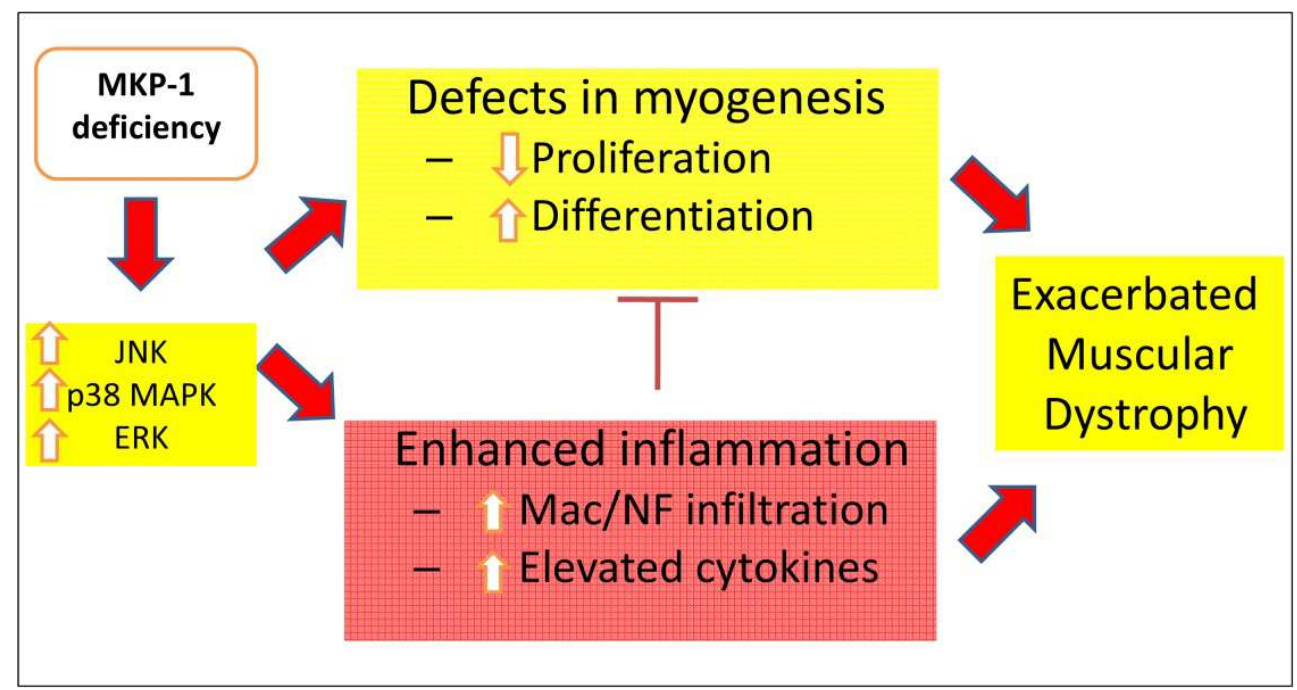

Fig. 2. MKP-1 and Duchenne Muscular Dystrophy. MKP-1 regulates both myoblast proliferation and differentiation (see Figure 1). Loss of MKP-1 in a mouse model of Duchennes' muscular dystrophy exacerbates the dystrophic phenotype due to enhanced MAPK activity, which inhibits myoblast proliferation, and ectopically enhances differentiation. In conjunction, MKP-1 has a profound effect in macrophages (Mac) and neutrophils (NF), which as a result of enhanced MAPK activity, in the absence of MKP-1 become hyper-responsive leading to increased inflammatory responses. Together, both the increased inflammatory response and dysfunctional myoblast proliferation and differentiation exacerbates the dystrophic phenotype.

a potential therapeutic target for the treatment of certain dystrophies. However, given the uncertain role played by JNK in skeletal muscle regeneration this target should be approached cautiously. A recent study shows that treating dystrophic mice with the free radical scavenger a-lipoic acid and L-carnitine improved muscular dystrophy with a concomitant repression of ERK1/2, JNK and p38 MAPK activation (Hnia et al., 2007). This is quite a provocative result since reactive oxygen species have been shown to inhibit the actions of certain protein tyrosine phosphatases through modification of the catalytic cysteine residue (Tonks, 2005). Therefore, treatment of dystrophic muscle with free radical scavengers would be predicted to ameliorate the loss of PTP activity, including MKP activity, resulting in increased inactivation of MAPKs. However, it is not yet clear whether the improved myopathy is caused by the decreased activation of a particular MAPK and/or a combination thereof. In an Emery-Dreyfuss muscular dystrophy mouse model, which lacks the inner nuclear membrane protein A-type lamins (LMNA), JNK and ERK1/2 are highly activated in heart tissue and cardiomycytes (Muchir et al., 2007). Inhibition of the ERK1/2 upstream kinase MEK by PD098059 improves cardiomyopathy in Lmna mutant knock-in mice (Muchir et al., 2009), implying that molecules in the ERK1/2 pathway have therapeutic potential for the treatment of human Emery-Dreyfuss muscular dystrophy and potentially related disorders. 
Although the MAPK/MKP pathway represents a potentially attractive therapeutic target to treat muscular dystrophy, it is a challenging one given the fact that the MAPK/MKP module is a universal pathway serving a number of common control points in the regulation of cell proliferation, differentiation, migration, and survival. The challenge will be to identify MAPK/MKP family members that exhibit signaling preferences to skeletal muscle with those functions further selectively controlling the appropriate physiological response in dystrophic skeletal muscle tissue. Given the importance of p38 MAPK in promoting regenerative myogenesis an attractive strategy could involve enhancing p38 MAPK activity so as to promote either satellite cell activation, proliferation and/or differentiation in dystrophic tissue. This could conceivably be achieved either through activation of p38 MAPK itself or through inhibition of the MKP that opposes the physiologically relevant pool of p38 MAPK in these cells. Clearly, significant gaps in our knowledge need to be filled in this area, nevertheless it is an important goal given the devastating nature of these skeletal muscle diseases that still lack a successful treatment.

\section{Acknowledgements}

A.M.B. is supported by the National Institutes of Health and H.S. by a grant from the Muscular Dystrophy Association.

\section{References}

Bennett, A.M., and N.K. Tonks. 1997. Regulation of distinct stages of skeletal muscle differentiation by mitogen-activated protein kinases. Science. 278:1288-1291.

Bhatnagar, S., and A. Kumar. 2010. Therapeutic targeting of signaling pathways in muscular dystrophy. J Mol Med (Berl). 88:155-166.

Black, B.L., and E.N. Olson. 1998. Transcriptional control of muscle development by myocyte enhancer factor-2 (MEF2) proteins. Annu Rev Cell Dev Biol. 14:167-196.

Bogoyevitch, M.A. 2006. The isoform-specific functions of the c-Jun N-terminal Kinases (JNKs): differences revealed by gene targeting. Bioessays. 28:923-934.

Bogoyevitch, M.A., and N.W. Court. 2004. Counting on mitogen-activated protein kinases-ERKs 3, 4, 5, 6, 7 and 8. Cell Signal. 16:1345-1354.

Boutros, T., E. Chevet, and P. Metrakos. 2008. Mitogen-activated protein (MAP) kinase/MAP kinase phosphatase regulation: roles in cell growth, death, and cancer. Pharmacol Rev. 60:261-310.

Briata, P., S.V. Forcales, M. Ponassi, G. Corte, C.Y. Chen, M. Karin, P.L. Puri, and R. Gherzi. 2005. p38-dependent phosphorylation of the mRNA decay-promoting factor KSRP controls the stability of select myogenic transcripts. Mol Cell. 20:891-903.

Camps, M., C. Chabert, M. Muda, U. Boschert, C. Gillieron, and S. Arkinstall. 1998a. Induction of the mitogen-activated protein kinase phosphatase MKP3 by nerve growth factor in differentiating PC12. FEBS Lett. 425:271-276.

Camps, M., A. Nichols, C. Gillieron, B. Antonsson, M. Muda, C. Chabert, U. Boschert, and S. Arkinstall. 1998b. Catalytic activation of the phosphatase MKP-3 by ERK2 mitogenactivated protein kinase. Science. 280:1262-1265.

Chi, H., S.P. Barry, R.J. Roth, J.J. Wu, E.A. Jones, A.M. Bennett, and R.A. Flavell. 2006. Dynamic regulation of pro- and anti-inflammatory cytokines by MAPK phosphatase 1 (MKP-1) in innate immune responses. Proc Natl Acad Sci U S A. 103:2274-2279. 
Christie, G.R., D.J. Williams, F. Macisaac, R.J. Dickinson, I. Rosewell, and S.M. Keyse. 2005. The dual-specificity protein phosphatase DUSP9/MKP-4 is essential for placental function but is not required for normal embryonic development. Mol Cell Biol. 25:8323-8333.

Cuevas, B.D., A.N. Abell, and G.L. Johnson. 2007. Role of mitogen-activated protein kinase kinase kinases in signal integration. Oncogene. 26:3159-3171.

Davies, K.E., and K.J. Nowak. 2006. Molecular mechanisms of muscular dystrophies: old and new players. Nat Rev Mol Cell Biol. 7:762-773.

Deconinck, N., and B. Dan. 2007. Pathophysiology of duchenne muscular dystrophy: current hypotheses. Pediatr Neurol. 36:1-7.

Dorfman, K., D. Carrasco, M. Gruda, C. Ryan, S.A. Lira, and R. Bravo. 1996. Disruption of the erp/mkp-1 gene does not affect mouse development: normal MAP kinase activity in ERP/MKP-1-deficient fibroblasts. Oncogene. 13:925-931.

Dorman, C.M., and S.E. Johnson. 1999. Activated Raf inhibits avian myogenesis through a MAPK-dependent mechanism. Oncogene. 18:5167-5176.

Field, C.J., C.A. Thomson, J.E. Van Aerde, A. Parrott, A. Euler, E. Lien, and M.T. Clandinin. 2000. Lower proportion of CD45R0+ cells and deficient interleukin-10 production by formula-fed infants, compared with human-fed, is corrected with supplementation of long-chain polyunsaturated fatty acids. J Pediatr Gastroenterol Nutr. 31:291-299.

Fornaro, M., P.M. Burch, W. Yang, L. Zhang, C.E. Hamilton, J.H. Kim, B.G. Neel, and A.M. Bennett. 2006. SHP-2 activates signaling of the nuclear factor of activated T cells to promote skeletal muscle growth. J Cell Biol. 175:87-97.

Gallo, R., M. Serafini, L. Castellani, G. Falcone, and S. Alema. 1999. Distinct effects of Rac1 on differentiation of primary avian myoblasts. Mol Biol Cell. 10:3137-3150.

Gillespie, M.A., F. Le Grand, A. Scime, S. Kuang, J. von Maltzahn, V. Seale, A. Cuenda, J.A. Ranish, and M.A. Rudnicki. 2009. p38-\{gamma\}-dependent gene silencing restricts entry into the myogenic differentiation program. J Cell Biol. 187:991-1005.

Gredinger, E., A.N. Gerber, Y. Tamir, S.J. Tapscott, and E. Bengal. 1998. Mitogen-activated protein kinase pathway is involved in the differentiation of muscle cells. J Biol Chem. 273:10436-10444.

Groom, L.A., A.A. Sneddon, D.R. Alessi, S. Dowd, and S.M. Keyse. 1996. Differential regulation of the MAP, SAP and RK/p38 kinases by Pyst1, a novel cytosolic dualspecificity phosphatase. Embo J. 15:3621-3632.

Hammer, M., J. Mages, H. Dietrich, A. Servatius, N. Howells, A.C. Cato, and R. Lang. 2006. Dual specificity phosphatase 1 (DUSP1) regulates a subset of LPS-induced genes and protects mice from lethal endotoxin shock. J Exp Med. 203:15-20.

Hnia, K., G. Hugon, F. Rivier, A. Masmoudi, J. Mercier, and D. Mornet. 2007. Modulation of p38 mitogen-activated protein kinase cascade and metalloproteinase activity in diaphragm muscle in response to free radical scavenger administration in dystrophin-deficient Mdx mice. Am J Pathol. 170:633-643.

Hutter, D., P. Chen, J. Barnes, and Y. Liu. 2000. Catalytic activation of mitogen-activated protein (MAP) kinase phosphatase-1 by binding to p38 MAP kinase: critical role of the p38 C-terminal domain in its negative regulation. Biochem J. $352 \mathrm{Pt}$ 1:155-163.

Ishibashi, T., D.P. Bottaro, P. Michieli, C.A. Kelley, and S.A. Aaronson. 1994. A novel dual specificity phosphatase induced by serum stimulation and heat shock. J Biol Chem. 269:29897-29902.

Johnson, G.L., and R. Lapadat. 2002. Mitogen-activated protein kinase pathways mediated by ERK, JNK, and p38 protein kinases. Science. 298:1911-1912. 
Jones, N.C., K.J. Tyner, L. Nibarger, H.M. Stanley, D.D. Cornelison, Y.V. Fedorov, and B.B. Olwin. 2005. The p38alpha/beta MAPK functions as a molecular switch to activate the quiescent satellite cell. J Cell Biol. 169:105-116.

Khurana, A., and C.S. Dey. 2004. Involvement of c-Jun N-terminal kinase activities in skeletal muscle differentiation. J Muscle Res Cell Motil. 25:645-655.

Kolodziejczyk, S.M., G.S. Walsh, K. Balazsi, P. Seale, J. Sandoz, A.M. Hierlihy, M.A. Rudnicki, J.S. Chamberlain, F.D. Miller, and L.A. Megeney. 2001. Activation of JNK1 contributes to dystrophic muscle pathogenesis. Curr Biol. 11:1278-1282.

Kondoh, K., K. Sunadome, and E. Nishida. 2007. Notch signaling suppresses p38 MAPK activity via induction of MKP-1 in myogenesis. J Biol Chem. 282:3058-3065.

Kontaridis, M.I., X. Liu, L. Zhang, and A.M. Bennett. 2002. Role of SHP-2 in fibroblast growth factor receptor-mediated suppression of myogenesis in $\mathrm{C} 2 \mathrm{C} 12$ myoblasts. Mol Cell Biol. 22:3875-3891.

Koyama, T., Y. Nakaoka, Y. Fujio, H. Hirota, K. Nishida, S. Sugiyama, K. Okamoto, K. Yamauchi-Takihara, M. Yoshimura, S. Mochizuki, M. Hori, T. Hirano, and N. Mochizuki. 2008. Interaction of scaffolding adaptor protein Gab1 with tyrosine phosphatase SHP2 negatively regulates IGF-I-dependent myogenic differentiation via the ERK1/2 signaling pathway. J Biol Chem. 283:24234-24244.

Kumar, A., N. Khandelwal, R. Malya, M.B. Reid, and A.M. Boriek. 2004. Loss of dystrophin causes aberrant mechanotransduction in skeletal muscle fibers. Faseb J. 18:102-113.

Lang, J.M., K.A. Esser, and E.E. Dupont-Versteegden. 2004. Altered activity of signaling pathways in diaphragm and tibialis anterior muscle of dystrophic mice. Exp Biol Med (Maywood). 229:503-511.

Lluis, F., E. Ballestar, M. Suelves, M. Esteller, and P. Munoz-Canoves. 2005. E47 phosphorylation by p38 MAPK promotes MyoD/E47 association and musclespecific gene transcription. Embo J. 24:974-984.

Meriane, M., S. Charrasse, F. Comunale, and C. Gauthier-Rouviere. 2002. Transforming growth factor beta activates Rac1 and Cdc42Hs GTPases and the JNK pathway in skeletal muscle cells. Biol Cell. 94:535-543.

Misra-Press, A., C.S. Rim, H. Yao, M.S. Roberson, and P.J. Stork. 1995. A novel mitogenactivated protein kinase phosphatase. Structure, expression, and regulation. J Biol Chem. 270:14587-14596.

Muchir, A., P. Pavlidis, V. Decostre, A.J. Herron, T. Arimura, G. Bonne, and H.J. Worman. 2007. Activation of MAPK pathways links LMNA mutations to cardiomyopathy in Emery-Dreifuss muscular dystrophy. J Clin Invest. 117:1282-1293.

Muchir, A., J. Shan, G. Bonne, S.E. Lehnart, and H.J. Worman. 2009. Inhibition of extracellular signal-regulated kinase signaling to prevent cardiomyopathy caused by mutation in the gene encoding A-type lamins. Hum Mol Genet. 18:241-247.

Muda, M., U. Boschert, A. Smith, B. Antonsson, C. Gillieron, C. Chabert, M. Camps, I. Martinou, A. Ashworth, and S. Arkinstall. 1997. Molecular cloning and functional characterization of a novel mitogen-activated protein kinase phosphatase, MKP-4. J Biol Chem. 272:5141-5151.

Murray, J., and J.M. Huss. 2011. Estrogen-related receptor \{alpha\} regulates skeletal myocyte differentiation via modulation of the ERK MAP kinase pathway. Am J Physiol Cell Physiol. 301:C630-645.

Nakamura, A., G.V. Harrod, and K.E. Davies. 2001. Activation of calcineurin and stress activated protein kinase/p38-mitogen activated protein kinase in hearts of utrophin-dystrophin knockout mice. Neuromuscul Disord. 11:251-259. 
Nakamura, A., K. Yoshida, S. Takeda, N. Dohi, and S. Ikeda. 2002. Progression of dystrophic features and activation of mitogen-activated protein kinases and calcineurin by physical exercise, in hearts of mdx mice. FEBS Lett. 520:18-24.

Nakamura, A., K. Yoshida, H. Ueda, S. Takeda, and S. Ikeda. 2005. Up-regulation of mitogen activated protein kinases in $\mathrm{mdx}$ skeletal muscle following chronic treadmill exercise. Biochim Biophys Acta. 1740:326-331.

Noguchi, T., R. Metz, L. Chen, M.G. Mattei, D. Carrasco, and R. Bravo. 1993. Structure, mapping, and expression of erp, a growth factor-inducible gene encoding a nontransmembrane protein tyrosine phosphatase, and effect of ERP on cell growth. Mol Cell Biol. 13:5195-5205.

Nunes-Xavier, C., C. Roma-Mateo, P. Rios, C. Tarrega, R. Cejudo-Marin, L. Tabernero, and R. Pulido. 2011. Dual-specificity MAP kinase phosphatases as targets of cancer treatment. In Anticancer Agents Med Chem. Vol. 11. 109-132.

Owens, D.M., and S.M. Keyse. 2007. Differential regulation of MAP kinase signalling by dual-specificity protein phosphatases. Oncogene. 26:3203-3213.

Pearson, G., F. Robinson, T. Beers Gibson, B.E. Xu, M. Karandikar, K. Berman, and M.H. Cobb. 2001. Mitogen-activated protein (MAP) kinase pathways: regulation and physiological functions. Endocr Rev. 22:153-183.

Perdiguero, E., V. Ruiz-Bonilla, L. Gresh, L. Hui, E. Ballestar, P. Sousa-Victor, B. Baeza-Raja, M. Jardi, A. Bosch-Comas, M. Esteller, C. Caelles, A.L. Serrano, E.F. Wagner, and P. Munoz-Canoves. 2007. Genetic analysis of p38 MAP kinases in myogenesis: fundamental role of p38alpha in abrogating myoblast proliferation. Embo J. 26:12451256.

Porter, J.D. 2000. Introduction to muscular dystrophy. Microsc Res Tech. 48:127-130.

Rando, T.A. 2001. The dystrophin-glycoprotein complex, cellular signaling, and the regulation of cell survival in the muscular dystrophies. Muscle Nerve. 24:1575-1594.

Rohan, P.J., P. Davis, C.A. Moskaluk, M. Kearns, H. Krutzsch, U. Siebenlist, and K. Kelly. 1993. PAC-1: a mitogen-induced nuclear protein tyrosine phosphatase. Science. 259:1763-1766.

Roth, R.J., A.M. Le, L. Zhang, M. Kahn, V.T. Samuel, G.I. Shulman, and A.M. Bennett. 2009. MAPK phosphatase-1 facilitates the loss of oxidative myofibers associated with obesity in mice. J Clin Invest. 119:3817-3829.

Salojin, K.V., I.B. Owusu, K.A. Millerchip, M. Potter, K.A. Platt, and T. Oravecz. 2006. Essential role of MAPK phosphatase-1 in the negative control of innate immune responses. J Immunol. 176:1899-1907.

Shi, H., E. Boadu, F. Mercan, A.M. Le, R.J. Flach, L. Zhang, K.J. Tyner, B.B. Olwin, and A.M. Bennett. 2010. MAP kinase phosphatase-1 deficiency impairs skeletal muscle regeneration and exacerbates muscular dystrophy. Faseb J. 24:2985-2997.

Shi, H., J.M. Scheffler, J.M. Pleitner, C. Zeng, S. Park, K.M. Hannon, A.L. Grant, and D.E. Gerrard. 2008. Modulation of skeletal muscle fiber type by mitogen-activated protein kinase signaling. Faseb J. 22:2990-3000.

Shi, H., J.M. Scheffler, C. Zeng, J.M. Pleitner, K.M. Hannon, A.L. Grant, and D.E. Gerrard. 2009. Mitogen-activated protein kinase signaling is necessary for the maintenance of skeletal muscle mass. Am J Physiol Cell Physiol. 296:C1040-1048.

Simone, C., S.V. Forcales, D.A. Hill, A.N. Imbalzano, L. Latella, and P.L. Puri. 2004. p38 pathway targets SWI-SNF chromatin-remodeling complex to muscle-specific loci. Nat Genet. 36:738-743. 
Slack, D.N., O.M. Seternes, M. Gabrielsen, and S.M. Keyse. 2001. Distinct binding determinants for ERK2/p38alpha and JNK map kinases mediate catalytic activation and substrate selectivity of map kinase phosphatase-1. J Biol Chem. 276:16491-16500.

Soulsby, M., and A.M. Bennett. 2009. Physiological signaling specificity by protein tyrosine phosphatases. Physiology (Bethesda). 24:281-289.

Spence, H.J., A.S. Dhillon, M. James, and S.J. Winder. 2004. Dystroglycan, a scaffold for the ERK-MAP kinase cascade. EMBO Rep. 5:484-489.

Suelves, M., F. Lluis, V. Ruiz, A.R. Nebreda, and P. Munoz-Canoves. 2004. Phosphorylation of MRF4 transactivation domain by p38 mediates repression of specific myogenic genes. Embo J. 23:365-375.

Sunadome, K., T. Yamamoto, M. Ebisuya, K. Kondoh, A. Sehara-Fujisawa, and E. Nishida. 2011. ERK5 regulates muscle cell fusion through Klf transcription factors. Dev Cell. 20:192-205.

Tedesco, F.S., A. Dellavalle, J. Diaz-Manera, G. Messina, and G. Cossu. 2010. Repairing skeletal muscle: regenerative potential of skeletal muscle stem cells. J Clin Invest. 120:11-19.

Tonks, N.K. 2005. Redox redux: revisiting PTPs and the control of cell signaling. Cell. 121:667-670.

Tonks, N.K. 2006. Protein tyrosine phosphatases: from genes, to function, to disease. Nat Rev Mol Cell Biol. 7:833-846.

Turjanski, A.G., J.P. Vaque, and J.S. Gutkind. 2007. MAP kinases and the control of nuclear events. Oncogene. 26:3240-3253.

Wagers, A.J., and I.M. Conboy. 2005. Cellular and molecular signatures of muscle regeneration: current concepts and controversies in adult myogenesis. Cell. 122:659-667.

Weyman, C.M., and A. Wolfman. 1998. Mitogen-activated protein kinase kinase (MEK) activity is required for inhibition of skeletal muscle differentiation by insulin-like growth factor 1 or fibroblast growth factor 2. Endocrinology. 139:1794-1800.

Whitmarsh, A.J. 2006. The JIP family of MAPK scaffold proteins. Biochem Soc Trans. 34:828-832.

Wu, J.J., R.J. Roth, E.J. Anderson, E.-G. Hong, M.-K. Lee, C.S. Choi, P.D. Neufer, G.I. Shulman, J.K. Kim, and A.M. Bennett. 2006. Mice lacking MAP kinase phosphatase1 have enhanced MAP kinase activity and resistance to diet-induced obesity. Cell Metab. 4:61-73.

Wu, J.J., L. Zhang, and A.M. Bennett. 2005. The noncatalytic amino terminus of mitogenactivated protein kinase phosphatase 1 directs nuclear targeting and serum response element transcriptional regulation. Mol Cell Biol. 25:4792-4803.

Yang, B., D. Jung, D. Motto, J. Meyer, G. Koretzky, and K.P. Campbell. 1995. SH3 domainmediated interaction of dystroglycan and Grb2. J Biol Chem. 270:11711-11714.

Yokoyama, T., K. Takano, A. Yoshida, F. Katada, P. Sun, T. Takenawa, T. Andoh, and T. Endo. 2007. DA-Raf1, a competent intrinsic dominant-negative antagonist of the Ras-ERK pathway, is required for myogenic differentiation. J Cell Biol. 177:781-793.

Zarubin, T., and J. Han. 2005. Activation and signaling of the p38 MAP kinase pathway. Cell Res. 15:11-18.

Zetser, A., E. Gredinger, and E. Bengal. 1999. p38 mitogen-activated protein kinase pathway promotes skeletal muscle differentiation. Participation of the Mef2c transcription factor. J Biol Chem. 274:5193-5200.

Zhao, Q., X. Wang, L.D. Nelin, Y. Yao, R. Matta, M.E. Manson, R.S. Baliga, X. Meng, C.V. Smith, J.A. Bauer, C.H. Chang, and Y. Liu. 2006. MAP kinase phosphatase 1 controls innate immune responses and suppresses endotoxic shock. J Exp Med. 203:131-140. 


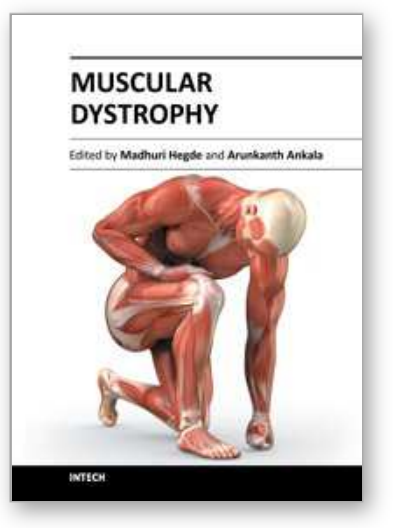

\author{
Muscular Dystrophy \\ Edited by Dr. Madhuri Hegde
}

ISBN 978-953-51-0603-6

Hard cover, 544 pages

Publisher InTech

Published online 09, May, 2012

Published in print edition May, 2012

With more than 30 different types and subtypes known and many more yet to be classified and characterized, muscular dystrophy is a highly heterogeneous group of inherited neuromuscular disorders. This book provides a comprehensive overview of the various types of muscular dystrophies, genes associated with each subtype, disease diagnosis, management as well as available treatment options. Though each different type and subtype of muscular dystrophy is associated with a different causative gene, the majority of them have overlapping clinical presentations, making molecular diagnosis inevitable for both disease diagnosis as well as patient management. This book discusses the currently available diagnostic approaches that have revolutionized clinical research. Pathophysiology of the different muscular dystrophies, multifaceted functions of the involved genes as well as efforts towards diagnosis and effective patient management, are also discussed. Adding value to the book are the included reports on ongoing studies that show a promise for future therapeutic strategies.

\title{
How to reference
}

In order to correctly reference this scholarly work, feel free to copy and paste the following:

Hao Shi and Anton M. Bennett (2012). Mitogen-Activated Protein Kinases and Mitogen-Activated Protein Kinase Phosphatases in Regenerative Myogenesis and Muscular Dystrophy, Muscular Dystrophy, Dr. Madhuri Hegde (Ed.), ISBN: 978-953-51-0603-6, InTech, Available from: http://www.intechopen.com/books/musculardystrophy/phosphatases-in-muscular-dystrophy

\section{INTECH}

open science | open minds

\section{InTech Europe}

University Campus STeP Ri

Slavka Krautzeka 83/A

51000 Rijeka, Croatia

Phone: +385 (51) 770447

Fax: +385 (51) 686166

www.intechopen.com

\section{InTech China}

Unit 405, Office Block, Hotel Equatorial Shanghai

No.65, Yan An Road (West), Shanghai, 200040, China

中国上海市延安西路65号上海国际贵都大饭店办公楼 405 单元

Phone: +86-21-62489820

Fax: $+86-21-62489821$ 
(C) 2012 The Author(s). Licensee IntechOpen. This is an open access article distributed under the terms of the Creative Commons Attribution 3.0 License, which permits unrestricted use, distribution, and reproduction in any medium, provided the original work is properly cited. 